\title{
The Application of Sodium-Glucose Cotransporter 2 Inhibitors to Chronic Kidney Disease Stage 4
}

\author{
Ayako Koguchi $^{\text {a, b}}$, Hiroki Adachi ${ }^{\text {a }}$, Hidekatsu Yanaia, b, c
}

\section{To the Editor}

Sodium-glucose cotransporter 2 inhibitors (SGLT2i) are reversible inhibitors of SGLT2, leading to reduction of renal glucose reabsorption and decrease of plasma glucose, in an insulin-independent manner [1]. Possible anti-atherosclerotic effects beyond glucose lowering of SGLT2 $\mathrm{i}$ can be predicted [2], and we previously showed that SGLT2i improve metabolic parameters including coronary risk factors $[3,4]$. Actually, the EMPA-REG OUTCOME trial that examined the effect of empagliflozin in addition to standard of care in patients with type 2 diabetes and established cardiovascular (CV) diseases demonstrated a significant reduction in the incidence of $\mathrm{CV}$ death and heart failure hospitalization [5]. Recently, the CANVAS program reported that canagliflozin significantly reduced hospitalization for heart failure as compared with placebo by $33 \%$ as well as empagliflozin [6]. Further, the CVD-REAL study, the large multinational study, presented that treatment with SGLT2 $i$ was associated with a lower risk of hospitalization for heart failure and death as compared with other glucose lowering drugs [7]. Prevention of development of heart failure by SGLT2i has been attracting a lot of attention [8].

SGLT2 is expressed in the proximal tubule of kidney and mediates reabsorption of approximately $90 \%$ of the filtered glucose load [9]; therefore, SGLT2i are not recommended for use in patients with renal insufficiency. Our previous studies demonstrated that estimated glomerular filtration rate (eGFR) has an influence on the improvement in $\operatorname{HbA1c}[10,11]$, and liver function by SGLT2i [10]. However, the EMPA-REG OUTCOME trial showed that empagliflozin significantly reduced incident or worsening of nephropathy, progression to macroalbuminuria, doubling of serum creatinine level accompanied by eGFR of $\leq 45 \mathrm{~mL} / \mathrm{min} / 1.73 \mathrm{~m}^{2}$ and initiation of renal replacement therapy [12]. The CANVAS program also demon-

\footnotetext{
Manuscript submitted October 5, 2017, accepted October 12, 2017

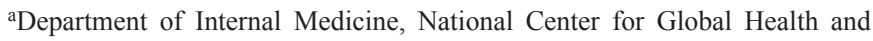
Medicine, Kohnodai Hospital, Chiba, Japan

${ }^{b}$ Department of Neurology, Center Hospital of the National Center for Global Health and Medicine, Tokyo, Japan

${ }^{\mathrm{c} C}$ Corresponding Author: Hidekatsu Yanai, Department of Internal Medicine, National Center for Global Health and Medicine, Kohnodai Hospital, 1-7-1 Kohnodai, Ichikawa, Chiba 272-8516, Japan.

Email: dyanai@hospk.ncgm.go.jp
}

doi: https://doi.org/10.14740/jocmr3220w strated that canagliflozin reduced progression to macroalbuminuria, and $40 \%$ reduction in eGFR, renal replacement therapy, or renal death [6]. Both the EMPA-REG OUTCOME trial and CANVAS program proposed the renal protective effect of SGLT2i. In the EMPA-REG OUTCOME trial, mean eGFR at baseline in low-eGFR group was almost $48 \mathrm{~mL} / \mathrm{min} / 1.73 \mathrm{~m}^{2}$, and the rate of patients with urinary albumin-to-creatinine ratio $>300$ was $18 \%$. In the CANVAS program, mean eGFR at baseline was $76 \mathrm{~mL} / \mathrm{min} / 1.73 \mathrm{~m}^{2}$, and the rate of patients with macroalbuminuria was $7.1 \%$. Effects of SGLT2i on renal function and proteinuria in patients with severe renal insufficiency such as chronic kidney diseases (CKD) stage 4 and nephrotic syndrome remain largely unknown.

Here, we present a 36-year-old man with a long-standing history of type 2 diabetes (21 years) admitted for pneumonia and heart failure. He had a significant prior history of obesity, and CKD stage 4 and nephrotic syndrome due to diabetic nephropathy. Dapagliflozin, one of SGLT2i, successfully reduced his urine protein and body weight, and improved heart failure and exercise tolerance without any adverse effects.

On admission, body height, weight and body mass index were $158.6 \mathrm{~cm}, 137.9 \mathrm{~kg}$ and $54.8 \mathrm{~kg} / \mathrm{m}^{2}$, respectively. Fever, tachycardia (heart rate, $118 / \mathrm{min})$, hypoxemia $\left(\mathrm{SpO}_{2} 94 \%\right.$ with 4 L oxygen) and tachypnea (respiratory rate, over 20/min) and abnormal findings of chest X-ray (cardiomegaly, pleural effusion and consolidation) suggested the existence of heart failure and pneumonia. Systolic and diastolic blood pressures were 168 and $95 \mathrm{~mm} \mathrm{Hg}$, respectively. His eGFR, serum creatinine, and daily urinary protein levels were $14 \mathrm{~mL} / \mathrm{min} / 1.73 \mathrm{~m}^{2}, 4.7$ $\mathrm{mg} / \mathrm{dL}$ and $15.5 \mathrm{~g} /$ day, suggesting the existence of CKD stage 4 and nephrotic syndrome [13]. At 3 weeks before admission, plasma glucose and HbA1c levels were $254 \mathrm{mg} / \mathrm{dL}$ and $8.3 \%$, respectively.

He had been treated by liraglutide $(0.9 \mathrm{mg} /$ day $)$, pioglitazone (15 mg/day), insulin aspart (40, 20 and $24 \mathrm{U}$ before breakfast, lunch and dinner, respectively) and insulin glargine (65 $\mathrm{U}$ at bedtime). We stopped the use of pioglitazone because of the development of heart failure, and started to use dapagliflozin ( $5 \mathrm{mg}$ /day) on day 4. Clinical course is shown in Figure 1. Body weight promptly decreased and body weight loss of over $20 \mathrm{~kg}$ was obtained during hospitalization. Serum creatinine gradually decreased after a transient increase, and eGFR also gradually increased after a transient decrease. Urinary protein promptly decreased, and a significant reduction (-10.8 $\mathrm{g}$ /day) was obtained during hospitalization.

Low plasma B-type natriuretic peptide (BNP) levels in obesity due to an increased clearance of active natriuretic 


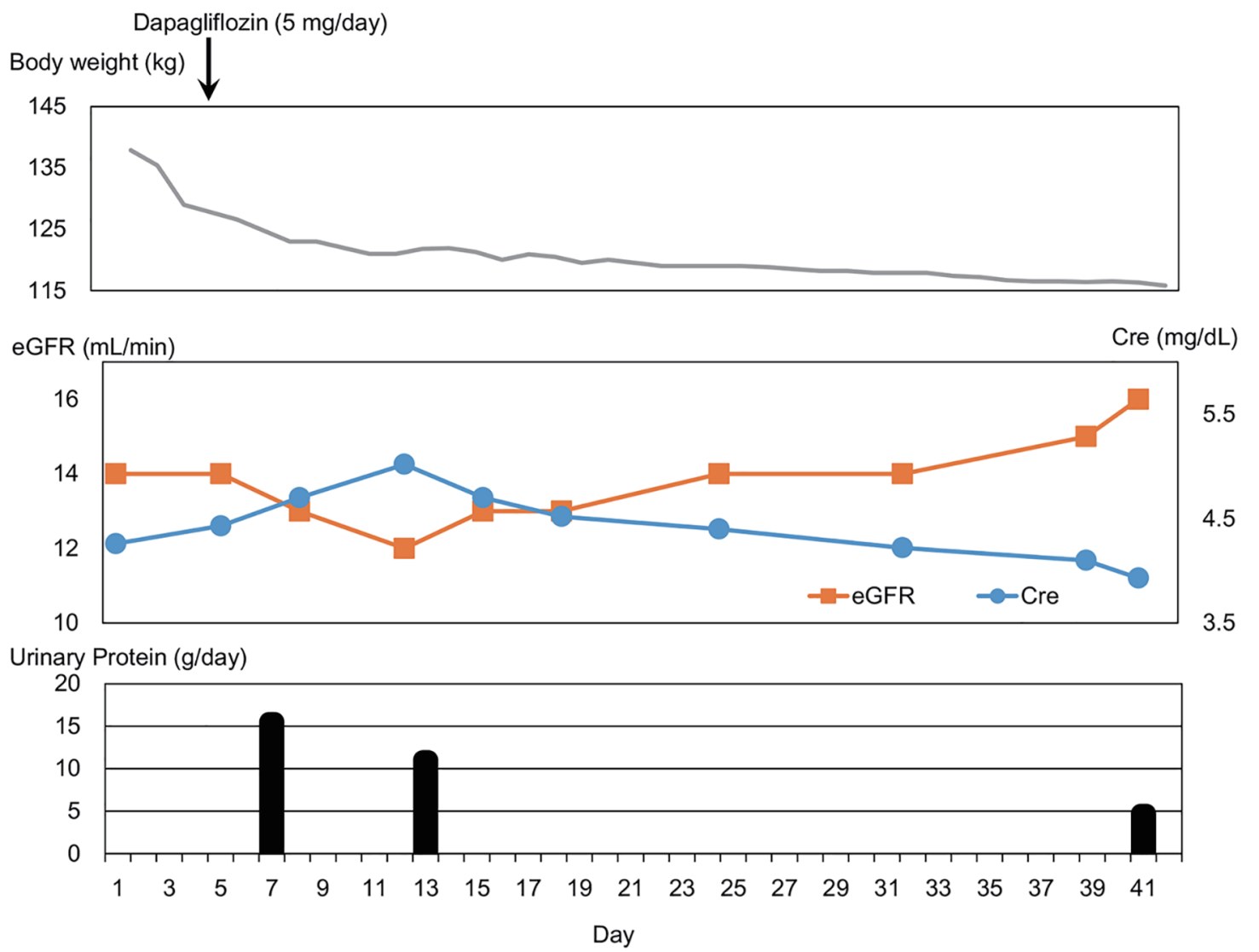

Figure 1. Clinical course. Cre: creatinine; eGFR: estimated glomerular filtration rate.

peptides by visceral fat was reported [14]. On admission, his plasma BNP level was high $(38.6 \mathrm{pg} / \mathrm{mL}$; normal range, 0 $18.4 \mathrm{pg} / \mathrm{mL}$ ) and his BNP increased from 7.9 to $38.6 \mathrm{pg} / \mathrm{mL}$ in 3 weeks before admission, supporting the development of heart failure. His plasma BNP level decreased to $5.3 \mathrm{pg} / \mathrm{mL}$ at 11 weeks after the start of dapagliflozin. At 4 days after the start of dapagliflozin, serum ketone bodies level was high (557 $\mu \mathrm{mol} / \mathrm{L}$; normal range, $<130 \mu \mathrm{mol} / \mathrm{L})$, and continued to be high $(407 \mu \mathrm{mol} / \mathrm{L}$; normal range, $<130 \mu \mathrm{mol} / \mathrm{L})$ until 39 days after the start of dapagliflozin. He was discharged from hospital at 40 days after the start of dapagliflozin. The treatment for his diabetes was changed to liraglutide $(0.9 \mathrm{mg} /$ day $)$, dapagliflozin ( $5 \mathrm{mg} /$ day), insulin aspart (46, 10 and $16 \mathrm{U}$ before break- fast, lunch and dinner, respectively) and insulin glargine (41 U at bedtime). Daily insulin dose decreased from 149 to $113 \mathrm{U}$.

To our knowledge, this is the first report to show the renal and cardiac protective effects of SGLT2 $\mathrm{i}$ in a type 2 diabetic patient complicated with CKD stage 4, nephrotic syndrome and heart failure. The addition of dapagliflozin remarkably reduced body weight, serum creatinine level and urinary protein, and increased eGFR. Possible mechanisms for renal protective effects of SGLT2i are shown in Table 1 [5, 15-21]. Increased serum ketone bodies, reduced blood pressure and amelioration of heart failure were observed in this patient. Various factors might contribute to the improvement of renal function.

In this patient, prompt body weight loss, increased exer-

Table 1. Possible Mechanisms for Renal Protective Effects of SGLT2 Inhibitors
1. Reduced blood pressure, arterial stiffness and vascular resistance $[15,16]$
2. Decrease in glomerular hyperfiltration [17]
3. Decrease in intraglomerular pressure [18]
4. Reduction in serum uric acid levels [5]
5. Utilization of ketone bodies by failing kidney/heart [19]
6. Improvement in tubulointerstitial hypoxia [20]
7. Increase of erythropoietin [21]
8. Improvement in renal fucntion due to improved cardiac function 
cise tolerance and reduced plasma BNP level were observed after the start of SGLT2i use, suggesting amelioration of heart failure due to SGLT2i. Reduced blood pressure, osmotic diuresis, and induction of utilization of ketone bodies by failing heart, due to SGLT2i, might contribute to amelioration of heart failure [19]. In addition to discontinuation of pioglitazone, reduced daily insulin dose $(-25 \%)$ and improved renal function, due to SGLT2i, might also contribute to the improvement of heart failure.

We have to mention the limitation of our observation. This is just a case report. To elucidate our hypothesis, further studies, preferably with larger numbers of subjects with CKD stage 4, will be needed.

In conclusion, the application of SGLT2 $\mathrm{i}$ might be a useful therapeutic option for CKD stage 4.

\section{References}

1. Jabbour SA, Goldstein BJ. Sodium glucose co-transporter 2 inhibitors: blocking renal tubular reabsorption of glucose to improve glycaemic control in patients with diabetes. Int J Clin Pract. 2008;62(8):1279-1284.

2. Yanai H, Katsuyama H, Hamasaki H, Adachi H, Moriyama S, Yoshikawa R, Sako A. Sodium-glucose cotransporter 2 inhibitors: possible anti-atherosclerotic effects beyond glucose lowering. J Clin Med Res. 2016;8(1):1014.

3. Katsuyama H, Hamasaki H, Adachi H, Moriyama S, Kawaguchi A, Sako A, Mishima S, et al. Effects of sodium-glucose cotransporter 2 inhibitors on metabolic parameters in patients with type 2 diabetes: a chart-based analysis. J Clin Med Res. 2016;8(3):237-243.

4. Yanai H, Hakoshima M, Adachi H, Kawaguchi A, Waragai Y, Harigae T, Masui Y, et al. Effects of six kinds of sodium-glucose cotransporter 2 inhibitors on metabolic parameters, and summarized effect and its correlations with baseline data. J Clin Med Res. 2017;9(7):605-612.

5. Zinman B, Wanner C, Lachin JM, Fitchett D, Bluhmki E, Hantel S, Mattheus M, et al. Empagliflozin, cardiovascular outcomes, and mortality in type 2 diabetes. N Engl J Med. 2015;373(22):2117-2128.

6. Neal B, Perkovic V, Mahaffey KW, de Zeeuw D, Fulcher G, Erondu N, Shaw W, et al. Canagliflozin and cardiovascular and renal events in type 2 diabetes. N Engl J Med. 2017;377(7):644-657.

7. Kosiborod M, Cavender MA, Fu AZ, Wilding JP, Khunti K, Holl RW, Norhammar A, et al. Lower risk of heart failure and death in patients initiated on sodium-glucose cotransporter-2 inhibitors versus other glucose-lowering drugs: The CVD-REAL study (comparative effectiveness of cardiovascular outcomes in new users of sodium-glucose cotransporter-2 inhibitors). Circulation. 2017;136(3):249-259.

8. Yanai H. Sodium-glucose cotransporter 2 inhibitors for heart failure. J Endocrinol Metab. 2017;7(3):75-76.
9. Vallon V, Platt KA, Cunard R, Schroth J, Whaley J, Thomson SC, Koepsell H, et al. SGLT2 mediates glucose reabsorption in the early proximal tubule. J Am Soc Nephrol. 2011;22(1):104-112.

10. Katsuyama H, Yanai H. An influence of the estimated glomerular filtration rate on improvement in metabolic parameters by sodium-glucose cotransporter 2 inhibitors. J Clin Med Res. 2016;8(6):486-488.

11. Yanai H, Hakoshima M, Adachi H. What properties of sodium-glucose cotransporter 2 inhibitors determine the improvement in hemoglobin A1c and body weight? J Clin Med Res. 2017;9(5):446-448.

12. Wanner C, Inzucchi SE, Lachin JM, Fitchett D, von Eynatten M, Mattheus M, Johansen OE, et al. Empagliflozin and progression of kidney disease in type 2 diabetes. $\mathrm{N}$ Engl J Med. 2016;375(4):323-334.

13. National Kidney F. K/DOQI clinical practice guidelines for chronic kidney disease: evaluation, classification, and stratification. Am J Kidney Dis. 2002;39(2 Suppl 1):S1266.

14. Clerico A, Giannoni A, Vittorini S, Emdin M. The paradox of low BNP levels in obesity. Heart Fail Rev. 2012;17(1):81-96.

15. Chilton R, Tikkanen I, Cannon CP, Crowe S, Woerle HJ, Broedl UC, Johansen OE. Effects of empagliflozin on blood pressure and markers of arterial stiffness and vascular resistance in patients with type 2 diabetes. Diabetes Obes Metab. 2015;17(12):1180-1193.

16. Cherney DZ, Perkins BA, Soleymanlou N, Har R, Fagan $\mathrm{N}$, Johansen OE, Woerle HJ, et al. The effect of empagliflozin on arterial stiffness and heart rate variability in subjects with uncomplicated type 1 diabetes mellitus. Cardiovasc Diabetol. 2014;13:28.

17. Vallon V, Gerasimova M, Rose MA, Masuda T, Satriano J, Mayoux E, Koepsell H, et al. SGLT2 inhibitor empagliflozin reduces renal growth and albuminuria in proportion to hyperglycemia and prevents glomerular hyperfiltration in diabetic Akita mice. Am J Physiol Renal Physiol. 2014;306(2):F194-204.

18. Skrtic M, Yang GK, Perkins BA, Soleymanlou N, Lytvyn Y, von Eynatten M, Woerle HJ, et al. Characterisation of glomerular haemodynamic responses to SGLT2 inhibition in patients with type 1 diabetes and renal hyperfiltration. Diabetologia. 2014;57(12):2599-2602.

19. Mudaliar S, Alloju S, Henry RR. Can a shift in fuel energetics explain the beneficial cardiorenal outcomes in the EMPA-REG OUTCOME study? A Unifying Hypothesis. Diabetes Care. 2016;39(7):1115-1122.

20. Sano M, Takei M, Shiraishi Y, Suzuki Y. Increased hematocrit during sodium-glucose cotransporter 2 inhibitor therapy indicates recovery of tubulointerstitial function in diabetic kidneys. J Clin Med Res. 2016;8(12):844-847.

21. Yanai H, Katsuyayama H. A possible mechanism for renoprotective effect of sodium-glucose cotransporter 2 inhibitor: elevation of erythropoietin production. J Clin Med Res. 2017;9(2):178-179. 